\title{
DETERMINATION OF ELECTROMECHANICAL TRANSIENT PROCESS CHARACTERISTICS USING EQUATION OF VARIABLES
}

تدنيد خصائص الحالات العابرة الكهروميكانيكية باستخدام دعادنة المنقيز ات

\section{J.I.Mansy}

Department of Electrical Engineering, Faculty of Engineering Mansoura University, Mansoura - Egypt.

ملخص إلبحث:

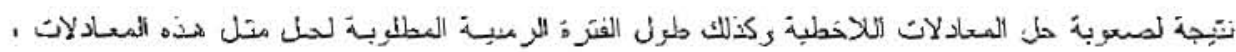

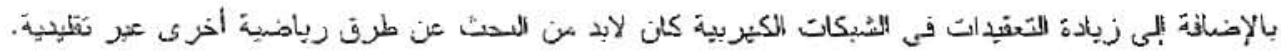

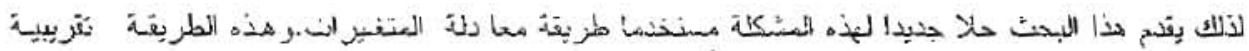

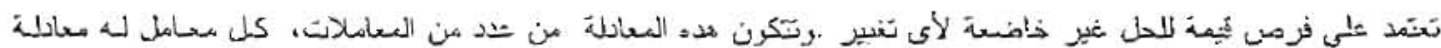

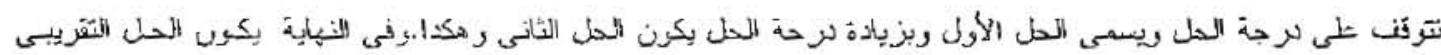

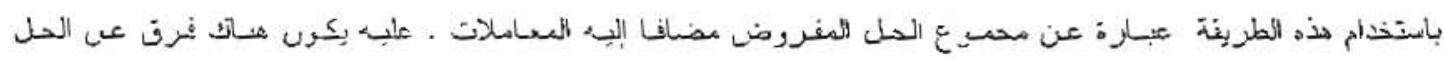

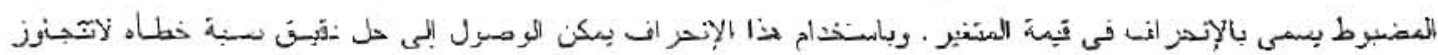

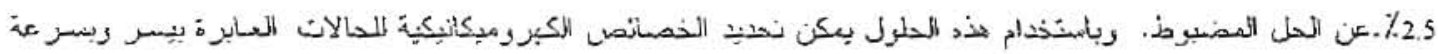
كبيز ة وبدثة عالهية.

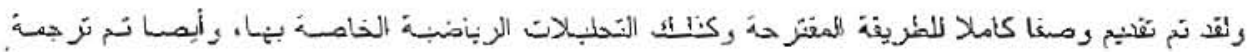

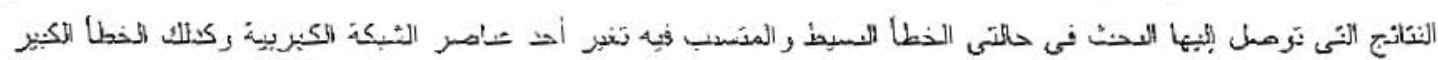

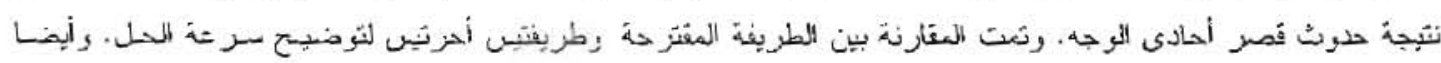

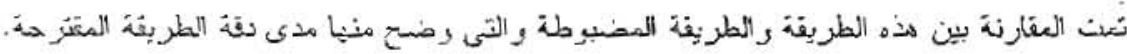

\section{ABSTRACT}

The equation of variables (EV) is an approximation of a mathematical method. ... is used to linearize noniinear equations. It is simple and accurate method and it offers quick solutions. This paper explains this equation and uses it to determine the characteristics of electromechanical transient process(EMTP) in electrical power systems.

The characteristics of EMTP are studied in this paper in two cases: first, simple disturbance, ( as a result of parameter variation) and second, large disturbance (as a result of occuring single S.C.) in this paper also. a comparison between the proposed method and another three methods is presented. One of these methods has exact solution and the others have an approximate one.

Accepted August 25/1996 


\section{INTRODUCTION}

Due to rapid growth of electrical power systems, over the past few years, stability analysis of the system became extremely difficult, even, using the present day computer facilities. Dynamic stability of electrical power systems stands as a subject of theoretical and practical interest.

Solution of non-linear equations, describing transient processes in power systems using method of optimal control theory directly, is too tidious to apply [1\&2].

The output of a conventional transient stability program is analyzed using transient energy functions for individuai machines [3].

The stability of power systems can be deduced from the roots of the system characteristic equation [4]. On the other hand, in a nonlinear system, such an inference is invalid [5] because its stability depends upon the initial conditions, as well as. the system parameters.

This paper illustrates the characteristics of EMTP using EV and presents the comparison between EV and those methods introduced in [6].

\section{ANALYSIS SOLUTION OF EV}

The error of determination EMTP characterestics with the help of EV may be evaluated. The solution of EV is analyzed in the case of selecting undisturbed and parameter variation system[6].Using this EV, the nonlinear differential equations will be transformed into second order linear differential equations with constant coefficients. These coefficients are illustrated and determined in [7].

As, in the capacity of parameter variation. the admittance (y) is considered. From [6 \& 8] the rotor angle $(\delta)$ is:

$\delta(t, y)=\delta^{*}+L_{1}(t) \Delta y+\frac{1}{2 !} L_{2}(t) \Delta y^{2}+\ldots .+R_{1}$,

where $\delta^{*}$ - the undisturbed value of $\delta$

$\mathrm{Ri}$ - the remainder of series (1)

$$
=L_{(i+1)}^{@}(t) \Delta y^{(i+1)} /(i+1) ! \ldots @ \varepsilon \Delta y=y-y^{*}
$$

$L_{1}(t), L_{2}(t)$ etc. are the first, second etc. solution of EV.

The first EV is presented in [6]. It has the following form:

$L_{1}(t)=C_{0}{ }^{(1)}\left(1-\cos \sqrt{a_{1}} t\right), . . C_{0}{ }^{(1)}=K_{0}{ }^{(1)} / a_{1}=-\tan \left(\delta^{\circ}\right) / y^{\circ}$ 
where

$$
\begin{aligned}
& a_{1}=\frac{2 \pi f_{0}}{T_{j}} E^{\prime} U^{*} y \cos \delta^{*}=\text { const. } \\
& K_{0}{ }^{(1)}=\frac{2 \pi f_{0}}{T_{j}} E^{\prime} U \sin \delta^{*}=\text { const. }
\end{aligned}
$$

$E^{\prime} \& U$-the generator and infinite bus voltages (in p. u.):

$T_{j}$ - the generator inertia constant:

$y^{*} \quad$ the undisturbed value of $y$ (in p.u.)

Analysis of EMTP characteristics is carried-out for the following cases:

i) Undisturbed stable(unstable) solution, standard solution, which is approximated with the help of variable coefficients method ( VCM) [6]:

ii) Undisturbed stable(unstabie) solution, standard solution unstable (stable).

The second EV is

$\ddot{\mathrm{L}}_{2}+\mathrm{a}_{1} \mathrm{~L}_{2}=\mathrm{d}_{2}(\mathrm{t}), \quad \mathrm{L}_{2}(0)=\dot{\mathrm{L}}_{2}(0)=0$

where

$$
d_{2}(t)=\frac{\partial^{2} f}{\partial \delta^{2}} L_{1}{ }^{2}(t)+2 \frac{\partial^{2} f}{\partial \delta \partial y} L_{j}(t)
$$

From Eq.(6) and Eq.(3)

$d_{2}=K_{0}^{(2)}+K_{1}^{(2)} \cos \sqrt{a_{1}} 1+K{ }_{2}^{(2)} \cos 2 \sqrt{a_{1}} t$

where; the upper index inficates the order of EV and the lower shows the ratio of argument.

From Eq.(6) and the initial conditions, $d_{2}(0)=0$. From this, we get,

$\mathrm{K}_{0^{(2)}}+\mathrm{K}_{1}^{(2)}+\mathrm{K}_{2}^{(2)}=0$

Integrating the second EV at the initial conditions and substituting in Eq.(7), we have

$L_{2}(t)=C_{0}^{(2)}+C_{1}^{(2)} \cos \sqrt{a_{1}} t+C_{2}^{(2)} \cos 2 \sqrt{a_{1}} t+R_{1}^{(2)} t \sin \sqrt{a_{1}} t$,

where $L_{2}(t)$ is determined from coefficients $k_{0}^{(1)}, k_{1}^{(2)}, k_{2}^{(2)}$ using the following expressions: 


$$
\begin{array}{ll}
C_{0}{ }^{(2)}=K_{0}{ }^{(2)} / \mathrm{a}_{1}, & \mathrm{C}_{1}{ }^{(2)}=-\left(\mathrm{C}_{0}{ }^{(2)}+\mathrm{C}_{2}{ }^{(2)}\right), \\
\mathrm{C}_{2}{ }^{(2)}=-\mathrm{K}_{2}{ }^{(2)} / 3 \mathrm{a}_{1}, & \mathrm{R}_{1}{ }^{(2)}=-\mathrm{K}_{1}{ }^{(2)} / 2 \sqrt{\mathrm{a}_{1}}
\end{array}
$$

For the third EV

$$
\ddot{\mathrm{L}}_{3}+\mathrm{a}_{1} \mathrm{~L}_{3}=\mathrm{d}_{3}(\mathrm{t}), \quad \mathrm{L}_{3}(0)=0 \text {, }
$$

where $d_{3}(t)$ is determined from the following formula:

$$
d_{3}(t)=\frac{\partial^{3} f}{\partial \delta^{3}} L_{1}{ }^{3}(t)+3 \frac{\partial^{3} f}{\partial \delta^{2} \partial y} L_{1}{ }^{2}(t)+3 \frac{\partial^{2} f}{\partial \delta^{2}} L_{1}(t) L_{2}(t)+3 \frac{\partial^{2} f}{\partial \delta \partial y} L_{2}(t)
$$

From Eqns.(12), (3) \& (9), we find

$$
\begin{aligned}
d_{3}(t)= & K_{0}{ }^{(3)} \div K_{1}{ }^{(3)} \cos \sqrt{a_{1}} t+K_{2}{ }^{(3)} \cos 2 \sqrt{a_{1}} i+K_{j}{ }^{(3)} \cos 3 \sqrt{a_{1}} t+ \\
& M_{1}{ }^{(3)}+t \sin \sqrt{a_{1}} t+M_{2}{ }^{(3)} t \sin 2 \sqrt{a_{1}} i
\end{aligned}
$$

From Eq. $(13)$ and initial conditions, $d_{3}(0)=0$. Then

$$
\mathrm{K}_{0}^{(3)}+\mathrm{K}_{1}^{(3)}+\mathrm{K}_{2}^{(3)}+\mathrm{K}_{3}^{(3)}=0
$$

Integrating Eq.(11) at initial conditions and substituting in Eq.(13), we have

$$
\begin{aligned}
L_{3}(t)= & C_{0}{ }^{(j)}+C_{1}{ }^{(\hat{j})} \cos \sqrt{a_{1}} t+C_{2}{ }^{(3)} \cos 2 \sqrt{a_{1}} t+C_{3}{ }^{(3)} \cos 3 \sqrt{a_{1}} t+ \\
& R_{1}{ }^{(3)} t \sin \sqrt{a_{1}} t+R_{2}{ }^{(j)} t \sin 2 \sqrt{a_{1}} t+S_{1}{ }^{(3)} t^{2} \cos \sqrt{a_{1}} t,
\end{aligned}
$$

where : $L_{3}(t)$ is determined from coefficients $k_{0}^{(3)}, k_{1}^{(3)}, k_{2}^{(3)} \cdot k_{3}^{(3)} \cdot M_{1}^{(3)} \cdot M_{2}^{(3)}$ using the following expressions:

$$
\begin{aligned}
& C_{0}{ }^{(3)}=\frac{K_{0}{ }^{(3)}}{a_{1}}, S_{1}^{(3)}=\frac{M_{1}^{(3)}}{4 \sqrt{a_{2}}}, R_{1}{ }^{(3)}=\frac{K_{1}{ }^{(3)}}{2 \sqrt{a_{1}}}+\frac{M_{1}^{(3)}}{4 a_{1}}, C_{3}{ }^{(3)}=\frac{K_{3}{ }^{(3)}}{8 a_{1}}, \\
& R_{2}{ }^{(3)}=\frac{M_{2}{ }^{(3)}}{3 a_{1}}, C_{2}^{(3)}=\frac{4 M_{2}{ }^{(3)}}{9 a_{1} \sqrt{a_{1}}}-\frac{k^{(3)}}{3 a_{1}}, C_{1}^{(3)}=-\left(C_{0}{ }^{(3)}+C_{2}{ }^{(3)}+C_{3}{ }^{(3)}\right)
\end{aligned}
$$

From the previous analysis, the solution of $i^{\text {th }}$ EV (general form) is possible to write in the form 


$$
\begin{aligned}
L_{i}(t)= & \sum_{0=0}^{i} C_{0}{ }^{(i)} \cos n \sqrt{a_{1} t}+t \sum_{0=0}^{i-1} R_{0}^{(i)} \sin n \sqrt{a_{1}} t+\ldots .+ \\
& t^{1-2} \sum_{n=0}^{i-1(-2)} Y_{n}{ }^{(i)} \cos n \sqrt{a_{1} t}+t^{i-i} \sum_{0=0}^{1-(1-1)} Z_{0}^{(i)} \sin n \sqrt{a_{1} t}
\end{aligned}
$$

$\mathrm{n}=0,1,2, \ldots \ldots, \mathrm{i}$

As follows from the obtained equations, the solutions of $E V L_{2}(t), \ldots \ldots, L_{1}(t)$ increase without bound on time increase. These solutions pass unbounded period of time through its initial value.

The performance of the proposed approach is investigated by computer simulation of power system consisting of a single machine connected to an infinite bus through long transmission line. The data and figure of the system are given in [1].

\section{EXAMPLE1:}

in this part, the obtained solutions of EV will be appijed on a mathematical example to show the character of approximated standard characterestics. Data of this example are $P_{0}=0.6$ p.u.; $E^{\prime}=1.2$ p.u. : $T_{j}=7$ s.; $\delta=26$ deg.; $y^{*}=1.14$ p.u. Coefficient vaiues of unbounded terms and obtained solutions of first three EV are listed in table 1. Solutions of first three EV depending on time are shown in Fig. 1. With the help of these solutions and the following formula

$\delta(t, y)=\delta^{0}+L_{1}(t) \Delta y+\frac{1}{2 !} L_{z}(t) \Delta^{2} y+\frac{1}{3 !} L_{3}(t) \Delta^{3} y$,

we shail consider three approximated standard characterestics EMTP: singie phase short circuit (S.C.) $y=0.34$ p.u.. $\Delta y=-0.8$ p.u (Fig.2); perturbation $y=0.82$ p.u. : $\Delta y=-0.32$ p.u.

(Fig.3); disturbance near to critical value for admittance $y_{c}=\frac{P_{0}}{E U} ; y=0.6$ p.u. $; \Delta y=-0.54$ p.u. (Fig.4). Curves in these figuresare obtained with the help of first, second and third EV. Also steady state undisturbed value of angle $\delta^{*}$ and standard characteristic $\delta_{0}(t)$.

On the basis of analyzed solutions EV, it is possible to describe the characteristics of EMTP. Thus, in the case of S.C.. calculate first three EV exact values in large range of angie variations with time (divergence larger than $5 \mathrm{deg}$. step at $0.58 \mathrm{~s}$. and angle vaiue $160 \mathrm{deg}$.).

After recovery of S.C. (i.e. parameter variation is decayed), EV and its solutions are varied. Besides initial conditions of these new EV. will be greater than zero( $\left.t=t_{\text {dis }}\right)$ where $t_{\text {dis }}$ is the removed fault time.

After decaying of parameter variations, the first EV have the following expressions: 


$$
\begin{array}{ll}
\ddot{L}_{1}{ }^{(0)}+a_{1} L_{1}{ }^{(0)}=0, & L_{1}{ }^{(0)}(\tau=0)=L_{1}\left(r=t_{d s}\right) \\
& \dot{L}_{1}{ }^{(0)}(\tau=0)=\dot{L}_{1}\left(t=r_{d s}\right)
\end{array}
$$

where $\tau=t-t_{\text {dis }}$; upper index $(0)$ is meaning that the solutions of EV are after recovery of S.C.

Solution of (17) has the following form

$L_{1}{ }^{(0)}(\tau)=A_{0}{ }^{(1)} \cos \left(\sqrt{a_{1}} \tau-\phi_{1}{ }^{(1)}\right)$

where, the upper index indicates identity value for ordier data of EV; and lower index shows the ratio of solution argument. Solution of amplitude values $\mathrm{A}_{0}^{(1)}$ and its angle $\phi_{1}^{(1)}$ depends on solution values of

$\ddot{L}_{1}+a_{l} L_{l}=d_{1}=k_{0}^{(1)} ; \quad L_{l}(0)=\dot{L}_{l}(0)=0$

and its first derivative at ending variation $m$ oment $\left(t_{\text {dis }}\right)$. These values are determined as:

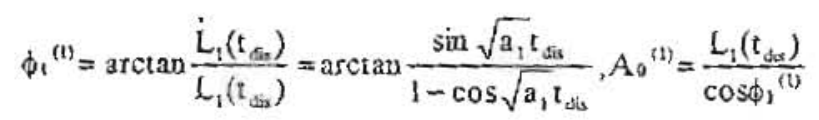

After a little manipulation in expression for solving first EV(18) it is possible to eliminate time $\tau$ as foliows

$L_{1}{ }^{(0)}(t)=A_{0}{ }^{(1)} \cos \left(\sqrt{a_{1}} t-\theta\right), \quad \theta\left(t_{d s}\right)=\sqrt{a_{i} t_{d s}}+\phi_{1}{ }^{(t)}$

Fig. 5 shows the relations between coefficient $L$ and time in the case of disconnected S.C. ( $t_{\text {dis }}=0.25$ s.). The solution of first EV is also shown in Fig. 5 " curve no. 1 ". This curve consists of two parts:first part represents Eq. (3) (from origin point to $t=t_{\text {dis }}$ ) and the second represents Eq.(18) (from $t>t_{\text {dis }}$ ). In Fig. 5. curve no.2 represents the same solution of EV without discon- necting S.C.. Bias quantity of extremal values of these two curves (1 \& 2 ) is equal to

$$
\Delta=\left(\pi-\phi 1^{(1)}\right) / \sqrt{a_{1}}
$$

Curve no.3 represents the solution in the case of $t=t_{\text {mdis }}=\sqrt{a_{1}}=0.4229 \mathrm{~s}$, at which extremal value of $L_{m}^{(1)}=2 C_{0}$, is reached.

For the second EV 


$$
\begin{aligned}
\ddot{L}_{2}^{(0)}+\mathrm{a}_{1} \mathrm{~L}_{2}^{(0)}=\mathrm{d}_{2}(\tau), & \mathrm{L}_{2}{ }^{(a)}(\tau=0)=\mathrm{L}_{2}\left(\mathrm{t}=\mathrm{t}_{\mathrm{c}_{3}}\right) \\
& \dot{\mathrm{L}}_{2}{ }^{(0)}(\tau=0)=\mathrm{L}_{2}\left(\tau=\mathrm{t}_{\mathrm{cos}}\right)
\end{aligned}
$$

where

$d_{2}(\tau)=\frac{\partial^{2} f}{\partial \delta^{2}} L_{1}{ }^{(0)^{3}}(\tau)=I_{0}^{(2)}+\mathrm{I}_{2 c^{2}}^{(2)} \cos 2 \sqrt{\mathrm{a}_{2}} \tau+\mathrm{I}_{22}{ }^{(2)} \sin 2 \sqrt{\mathrm{a}_{1}} \tau$

The right hand side of Eq. (22) is accomplished with the substitution of solution in Eq.(18). Integrating the second EV at given initial conditions and using Eq.(22), we obtain

$$
\mathrm{L}_{2}{ }^{(0)}(\tau)=\mathrm{A}_{0}{ }^{(2)}+\mathrm{A}_{1}{ }^{(2)} \cos \left(\sqrt{\mathrm{a}_{1}} \tau-\phi_{1}{ }^{(2)}\right)+\mathrm{A}_{2}{ }^{(2)} \cos \left(2 \sqrt{\mathrm{a}_{1}} \tau-\phi_{2}{ }^{(2)}\right)
$$

The solution of amplitudes and phases of Eq. (23) are determined through coefficients $1_{0}^{(2)}, 1_{2 \mathrm{c}}^{(2)}, \mathrm{l}_{2 \mathrm{~s}}^{(2)}$ as follows:

$$
\begin{aligned}
& A_{0}{ }^{(2)}=\frac{l_{0}{ }^{(2)}}{a_{1}} \cdot \phi_{1}^{(2)}=\arctan \frac{L_{2}\left(t_{d i s}\right)+2 l_{2 x}{ }^{(2)} / 3 \sqrt{a_{1}}}{L_{2}\left(t_{d+s}\right) \sqrt{a_{1}-\left(l_{0}{ }^{(2)}-l_{25}{ }^{(2)} / 3\right) / \sqrt{a_{1}}}} \\
& \phi:^{(2)}=\arctan \frac{l_{2 s^{(2)}}}{l_{2 e}{ }^{(2)}}, A_{1}^{(2)}=\left[L_{2}\left(t_{d i s}\right)-\left(l_{0}{ }^{(2)}-l_{2 e}{ }^{(2)} / 3\right)\right] / \cos \phi_{1}{ }^{(2)} \text {; } \\
& A_{2}{ }^{(2)}=l_{2 c}{ }^{(2)} /\left(3 a_{1} \cos \phi z^{(2)}\right)
\end{aligned}
$$

The solution of $i^{\text {th }}$ EV after disconnecting S.C. may be written as foliows:

$$
\begin{aligned}
& L_{i}^{(0)}(\tau)=A_{v}{ }^{(i)}+\sum_{n=1}^{i} A_{n}^{(i)} \cos \left(n \sqrt{a_{1}} \tau-\phi_{n}^{(i)}\right\}+\tau \sum_{n=1}^{i} Q_{n-2}{ }^{(i)} \cos \left[(n-2) \sqrt{a_{1}} \tau-\phi_{n-2 Q^{(i)}}\right]+
\end{aligned}
$$

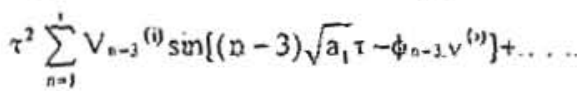

where $n=1,2, \ldots, i$.

The final admittance variations for $i^{\text {th }}$ solution of $j^{\text {th }}$ EV are

$L_{;}=\left\{\begin{array}{l}L_{j}(t), 0<t<t_{d x}, \quad \text { asin Eq. }(16) \\ L_{i}\left(t_{d i s}\right)=L_{1}{ }^{(0 ;}(\tau=0), t=t_{d u} \\ L_{,}{ }^{(0)}(\tau), t_{d i s}<t<t_{s}, \tau=t-t_{d s} \text { asin Eq. }(25)\end{array}\right.$

Notice that the obtained expressions are possibie to apply by considering a series number of iterations.Corresponding initial conditions are very necessary to be computed continuously. 


\section{EXAMPLE 2:}

In this section, consider a numerical example to indicate the behaviour of approximate characteristics after decaying of parameter variations. We used the same data in the previous example. Fig. 6 shows the solutions $L_{1}, L_{2} \& L_{3}$, which are determined from Eq. (26), at $t_{d i s}$ $=0.25 \mathrm{~s}$.. From this figure, we notice that the curves from origin point to $t=t_{d i s}$ are as in Fig. 1 . Amplitude and phase values of corresponding solutions, which are determined from Eqns.(19), (24),.., are given in table 2.

Fig.7 \& Fig.8 show approximate curves(time interval 0.05 s) $\delta_{0}$ with the help of $L_{1}, L_{2} \& L_{3}$ (curves 1,223). Fig.7 shows the disconnecting single phase S.C. process $(y=0.34 p . u, \Delta y=-0.8$ p.u.) within 0.25 s., but Fig. 8 shows finishing parameter variations $(y=0.82$ p.u., $\Delta y=-0.32$ p.u. ) within $0.25 \mathrm{~s}$. Also, these figures show the undisturbed angie value $\delta^{*}$.

A comparison between the proposed and exact solutions of the problem under study in the case of system parameter variation is shown in Fig. 9 This figure shows that the error of the proposed solution may be neglected.

\section{FINAL RESULTS ANALYSIS:}

As we conclude from figures, the obtained analytical solutions Eq.(26) permit, steady-state solutions give good approximate characterestics EMTP at disconnecting S.C. (finished admittance variations). In addition. in the case of small variations, curves $\mathrm{L}_{2}, \mathrm{~L}_{3}$ practically coinside with standard curve in Fig.8. But in the case of S.C. Sequence of using EV may achieve an accuracy near to standard characteristics $\hat{\delta}_{0}(\mathrm{t})$.

An approach to obtain the family of EMTP characteristics with the help of corresponding EV analytical solutions, at steady-state undisturbed values, have series of advantages in comparison with the other methods.

As shown from table 3, the proposed method solves the example under study in $1.5 \mathrm{sec}$. Also, the method is very fast compared with the other methods.

\section{CONCLUSIONS:}

From the previous analysis, figures and results, we conclude the following:-

1-With the help of EV solutions, characteristics family may be rapidly obtained.Rapidity of this method is greater than interval sequence method (nearly 10 times) and from 20 to 25 times greater than conventional method in $[6]$.

2-in the case of steady-state, undisturbed values can describe undisturbed transient process.

3- This method is simple, responding and accurate.

4- This method is applied on a simple electrical system ( generator with infinite bus.).

5- Comparing the results of this method with the exact solution, we find that the error of this method may be neglected. 
TaD! 1

\begin{tabular}{|c|c|c|c|c|c|c|c|}
\hline 1 & $k_{0}^{16}$ & $k_{i}^{\prime i}$ & $x^{1: 2}$ & $k_{s}^{61}$ & $\mathrm{~N}_{1}^{6:}$ & \multicolumn{2}{|l|}{$\mathrm{N}_{2}^{6: 1}$} \\
\hline $\begin{array}{l}1 \\
2 \\
3\end{array}$ & $\begin{array}{r}-1352.7 \\
2796.5 \\
-9484.8\end{array}$ & $\begin{array}{r}-2937.7 \\
20178.7\end{array}$ & $\begin{array}{r}143.13 \\
-707.9\end{array}$ & 2.7 .9 & -35622.4 & $-3<24$ & .7 \\
\hline$i$ & $\mathrm{C}_{0}^{111}$ & $c_{3}^{111}$ & $\mathrm{c}_{2}^{i^{i}}$ & $c_{3}^{i 2}$ & $\mathrm{R}^{6}=1$ & $2_{2}^{6}$ & $s_{1}^{163}$ \\
\hline$\left\{\begin{array}{l}1 \\
2 \\
3\end{array}\right.$ & $\begin{array}{r}-24.51 \\
50.68 \\
-171.9\end{array}$ & $\begin{array}{c}-49.83 \\
164.21\end{array}$ & $\begin{array}{r}-0.85 \\
7.91\end{array}$ & -0.13 & $\begin{array}{c}-198.19 \\
640.4\end{array}$ & 20.72 & -2299.1 \\
\hline
\end{tabular}

Trble 2

\begin{tabular}{|c|c|c|c|c|c|c|c|c|c|}
\hline i & $A_{0}^{(i)}$ & $A_{1}^{(i)}$ & $A_{2}^{123}$ & $\lambda_{3}^{1+1}$ & $Q_{1}^{213}$ & $\phi_{1}^{(i)}$ & $\phi_{2}^{\prime}=1$ & $\phi_{3}^{1+1}$ & $\varphi_{10}^{\prime i j}$ \\
\hline $\begin{array}{l}2 \\
2 \\
3\end{array}$ & $\begin{array}{r}-39.25 \\
6.56 \\
-16.73\end{array}$ & $\begin{array}{l}41.31 \\
38.51\end{array}$ & $\begin{array}{l}-2.29 \\
-5.9\end{array}$ & -0.45 & $72.2:$ & \begin{tabular}{|c|}
36.6 \\
-72.54 \\
65.4
\end{tabular} & $\begin{array}{r}-73.55 \\
70.25\end{array}$ & 70.35 & 53.19 \\
\hline
\end{tabular}

T上ప 0

Exicution time comperison for the proposid method and ozien nothods

\begin{tabular}{|c|c|}
\hline Mezhoss & Time in ssc. \\
\hline 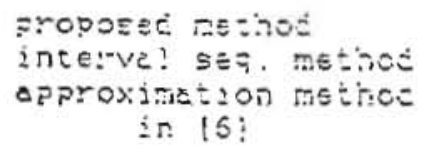 & $\begin{array}{l}-35 \\
24.53 \\
33.52\end{array}$ \\
\hline
\end{tabular}


E. 10 LII.Mansy

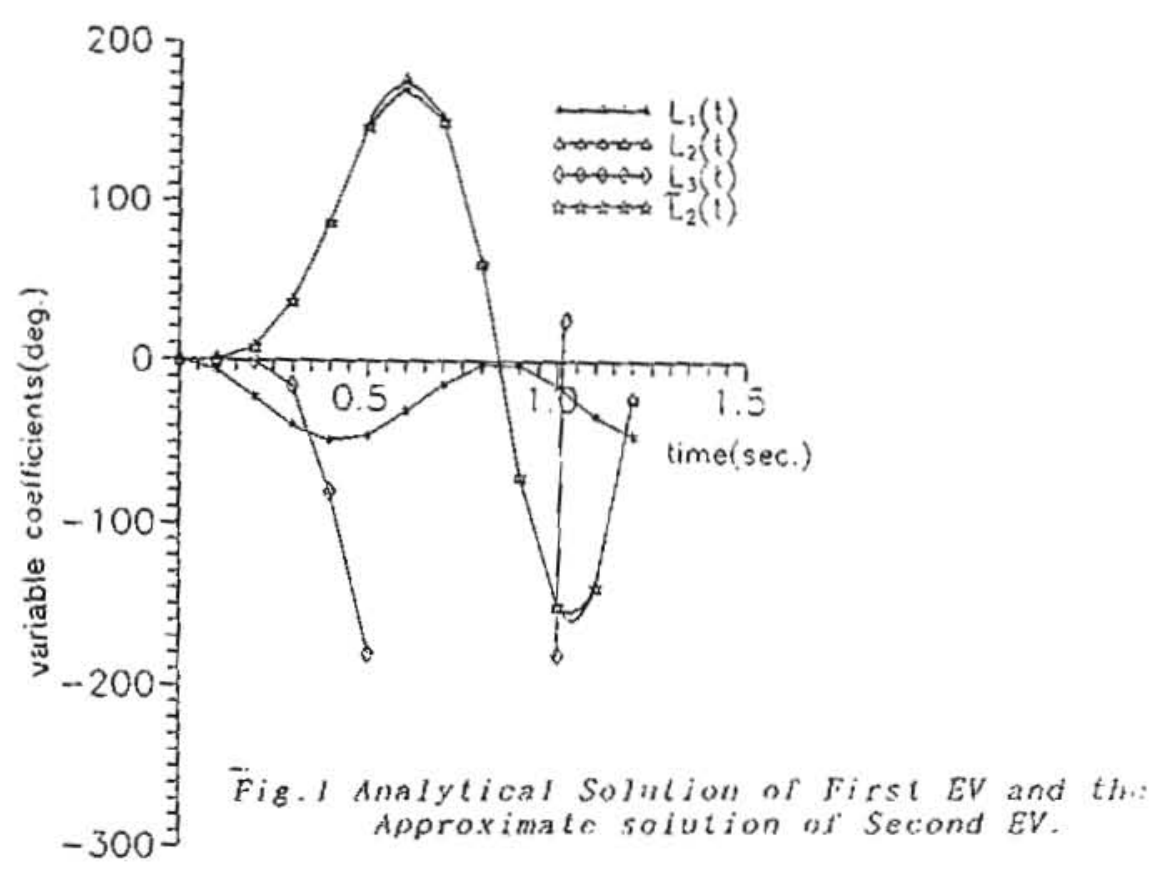

$\underbrace{200}_{10}$

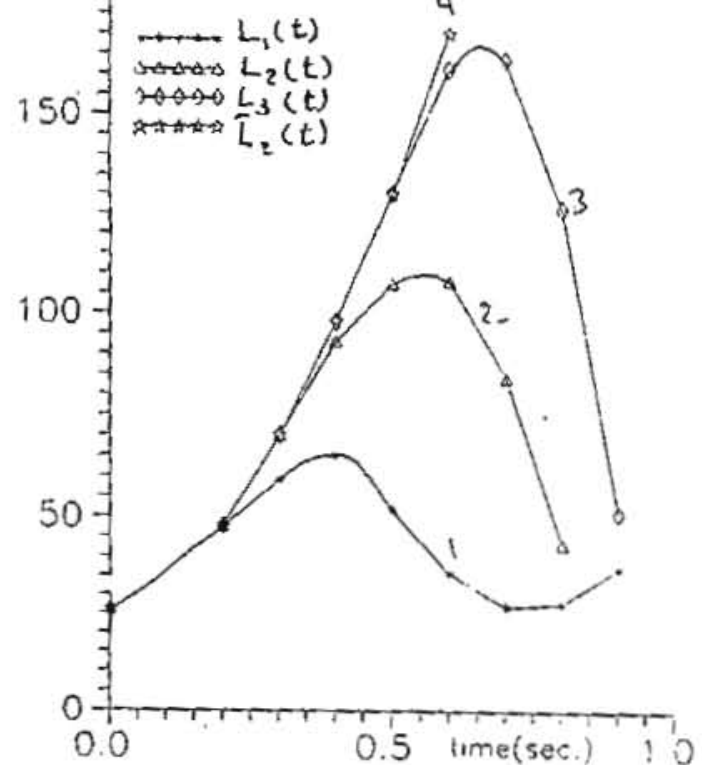

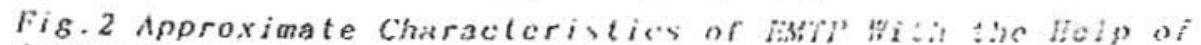

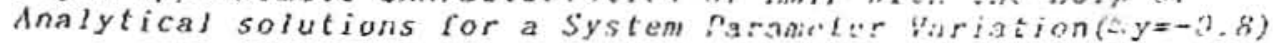




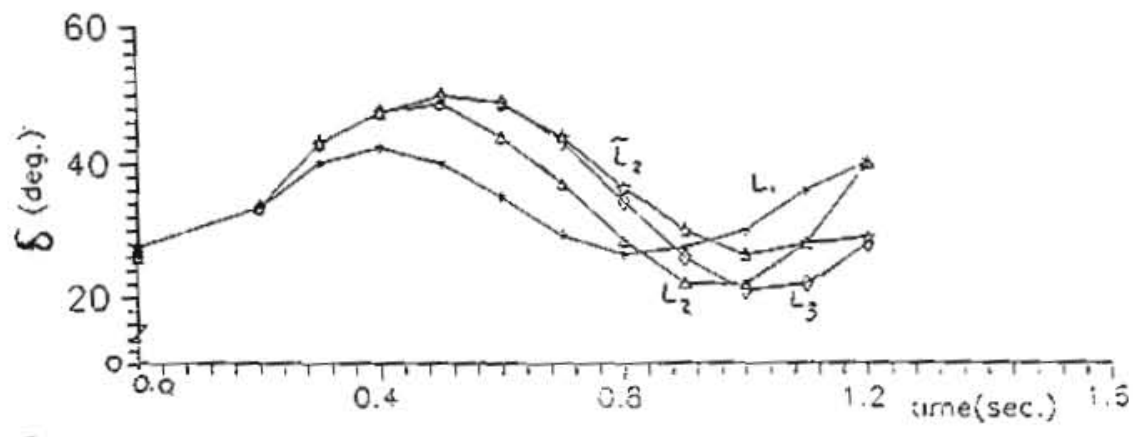

Fig. 3 Approximate Characterislics of EMT: With the lie if of Analytical solution: for a system Parameter Vartation( $y=-0.32)$

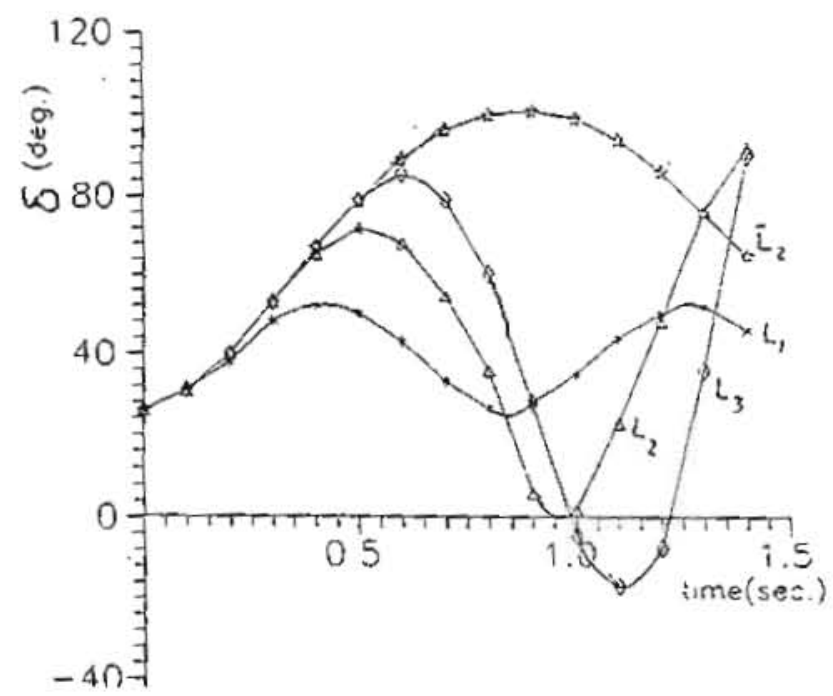

Fig. 4 Approximate Characteristics of EllTP Hith the lielp of Analytical solutiuns ior a Systcm Parameter Virzaiton(A $y=-0.54)$ 

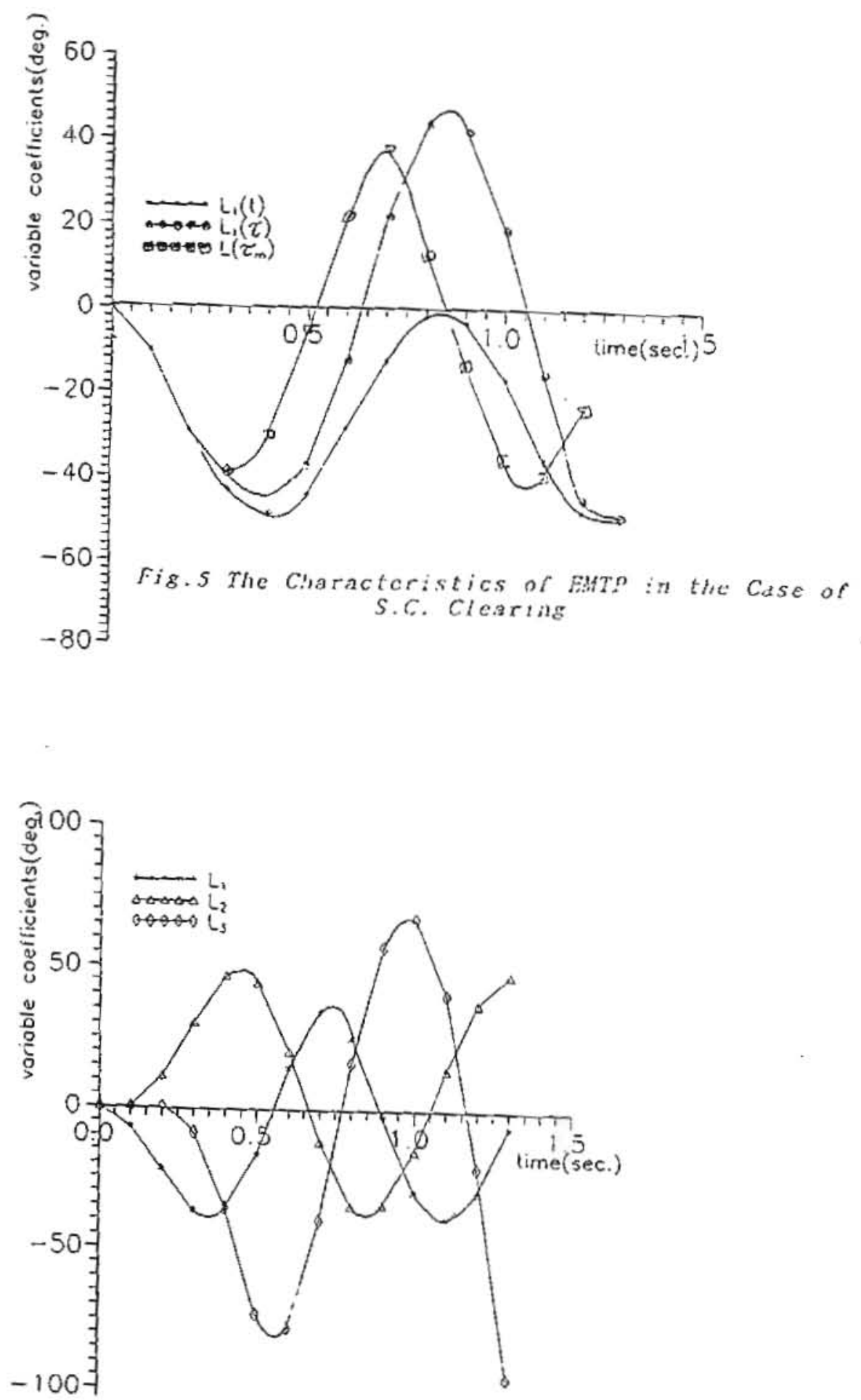

Fig. G Analytical Solution of Yirs: IV at Disconnecting S.C. 

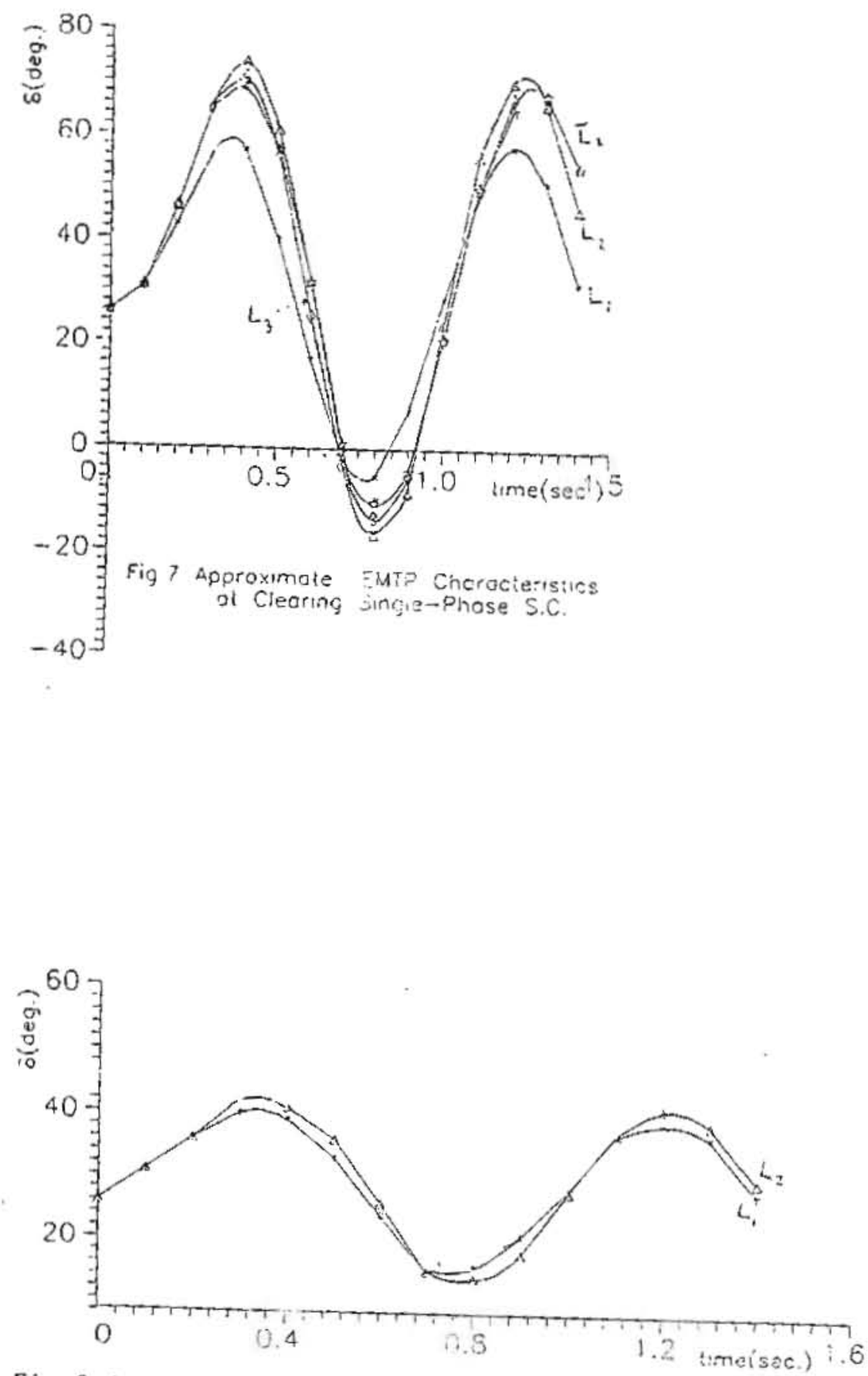

Fig. 8 Approximate EMTP Characteristics followne rowoval of Parameter Variations 


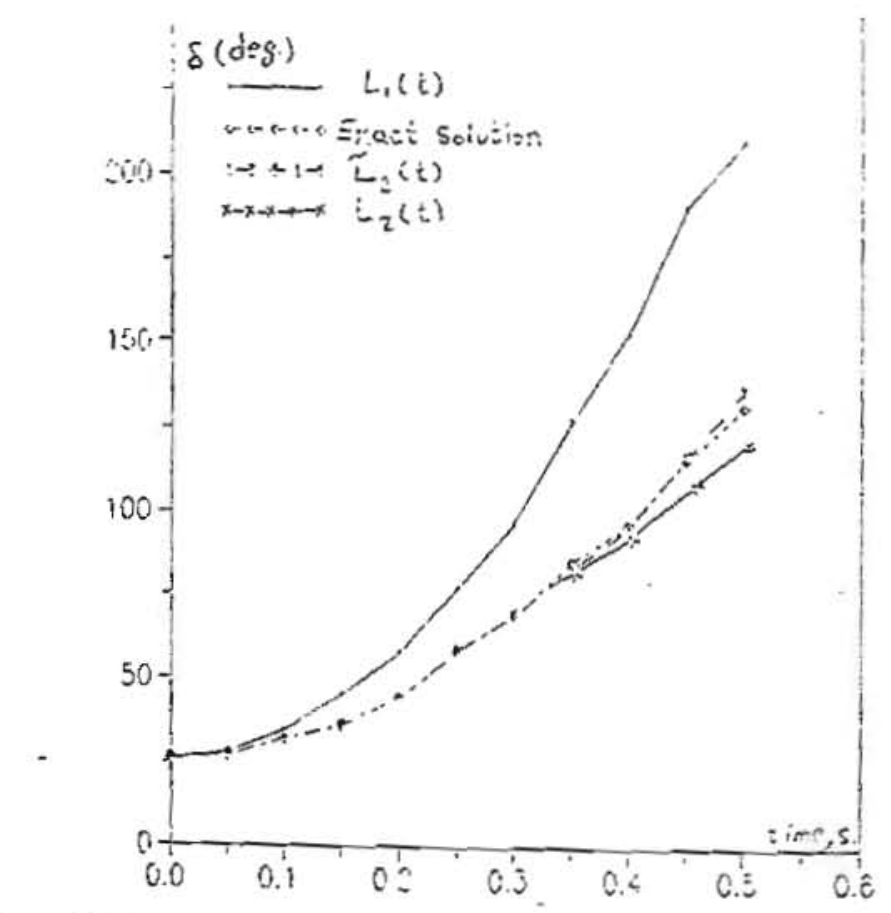

Fig. 9 Approvimale and Fixact characteristics of EllT?

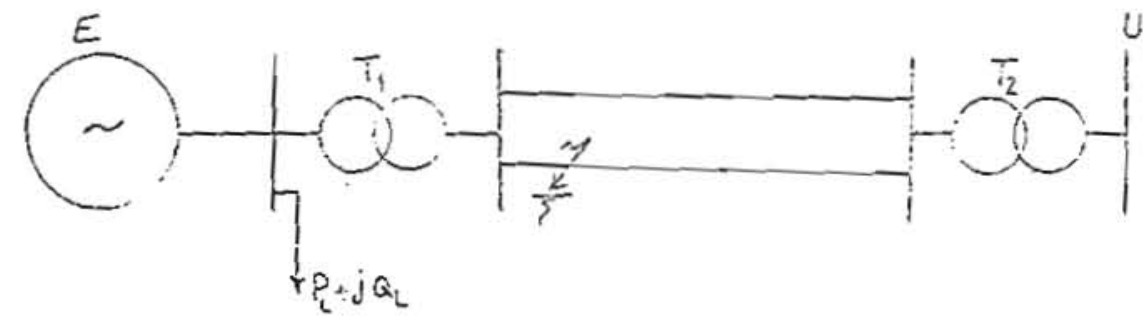

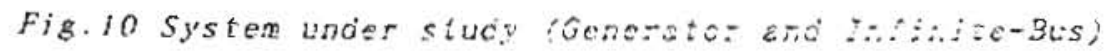




\section{REFERENCES:}

1- F.F. Areed and others "Optimal Control of Electrical Power Systems Using MaximalPrinciple." $13^{\text {th }}$ Internaticnal Congress for Statistics, Computer Science, Social and Demographic Research,March,1988, pp. 215-228.

2- V.A. Venikov"Electromechanical Transient Process in Electrical systems.".Higher School, 1985, $415 \mathrm{p}$.

3- A.A. Fouad and others"Transient Stability Program Output Analysis"IEEE Trans. on Power Systems.Vol.PWRS-1,No.1,Feb.,1986,pp. 2-9.

4- I.I.I. Mansy"Predicting the Transient Stability of Ceps Using AES Method."Mansoura Engineering Journal (MEJ), Vol.14,No.2, Dec.,1989, pp. E.30-E.39.

5- H.W. Dommel and N. Sato "Fast Transient Stability Solutions", IEEE Trans. Apparatus and Systems,Vol.PAS-86,July/Aug., 1972.pp.253-260.

6- 1.I.I. Mansy "Method Development for Determining Control Actions, Which Improve Transient Processes in Egyptian Power System." PHD Thesis, 1985.

7- I.I.I. Mansy and A.E.E. Ibrahim"Stability Analysis of Power System Using DSM",Mansoura Engineering Journal(MEJ), Vol.14.No.2,Dec.1989, pp. E.51 -E.62.

8- 1.1.1. Mansy and A.M.M. Aly"Electromechanical Transient Processe Analysis In Electrical Systems With the Help of Equation in Variations.", $19^{\text {th }}$ International Conference on Statistics,Computer Science and Applications, April , 1994, pp.160-171. 